\title{
Hydrodynamics and collective behaviour in relativistic nuclear collisions*
}

\author{
Dirk H. Rischke ${ }^{a}$ \\ a Physics Department, Pupin Physics Laboratories, Columbia University, \\ 538W 120th Street, New York, NY 10027, U.S.A.
}

\begin{abstract}
Hydrodynamics is applied to describe the dynamics of relativistic heavy-ion collisions. The focus of the present study is the influence of a possible (phase) transition to the quark-gluon plasma in the nuclear matter equation of state on collective observables, such as the lifetime of the system and the transverse directed flow of matter. It is shown that such a transition leads to a softening of the equation of state, and consequently to a time-delayed expansion which is in principle observable via two-particle correlation functions. Moreover, the delayed expansion leads to a local minimum in the excitation function of transverse directed flow around AGS energies.
\end{abstract}

\section{INTRODUCTION}

Lattice calculations of the thermodynamical functions of quantum chromodynamics (QCD) indicate [1] that, (at zero net baryon density) in the vicinity of a critical temperature $T_{c} \sim 160 \mathrm{MeV}$, strongly interacting matter undergoes a rapid transition from a (chirally broken, confined) hadronic phase to a (chirally symmetric, deconfined) quarkgluon plasma (QGP). The width $\Delta T$ of that transition region is presently only known to be in the range $0 \leq \Delta T<0.1 T_{c} \sim 16 \mathrm{MeV}$. Therefore, one cannot yet conclude whether the transition is a first order phase transition $(\Delta T=0)$, or merely a rapid increase of the entropy density associated with the change from $d_{H}$ hadronic to $d_{Q}$ quark and gluon degrees of freedom.

One of the primary goals of present relativistic heavy-ion physics is the creation and experimental observation of the predicted QGP phase of matter. Many signatures have been proposed such as electromagnetic radiation of thermal dileptons and photons [2], $J / \Psi$-suppression [3], jet quenching [4], strangelet formation [5], or disordered chiral condensates (DCC's) 6]. These signatures, however, do not depend directly on the actual form of the nuclear matter equation of state (EoS). Thermal electromagnetic radiation is, for instance, generic to any hot system, independent from its degrees of freedom (as long as they have electromagnetic charge). For example, it was shown [7] that a hot hadron gas shines as brightly as a QGP. Similarly, jet quenching and $J / \Psi$-suppression 8 are generic consequences of final state interactions in any form of dense matter [9]. Finally, strangelet

\footnotetext{
*This work was supported by the Director, Office of Energy Research, Division of Nuclear Physics of the Office of High Energy and Nuclear Physics of the U.S. Department of Energy under Contract No. DE-FG-02-93ER-40764.
} 
or DCC formation require very specific assumptions about the dynamical evolution of the system.

It is therefore of interest to study signals that are more directly related to the QCD equation of state. Signals of this type emerge from the influence of the EoS on the collective dynamical evolution of the system. Relativistic hydrodynamics [10] is the most suitable approach to study these signals, since it is the only dynamical model which provides a direct link between collective observables and the EoS. Of course, the use of such an approach requires a strong dynamical assumption, namely that the equilibration rates are much larger than typical gradients of thermodynamic quantities in the system. At least for high energy density QCD matter, radiative gluon energy loss was estimated to be sufficiently large [11] to support local equilibration on time scales less than $1 \mathrm{fm} / \mathrm{c}$. In the following, I therefore neglect dissipative effects and assume the validity of ideal hydrodynamics to compute the collective evolution of the system.

It was shown [12 [15] that the transition to the QGP softens the EoS in the transition region, and thus reduces the tendency of matter to expand on account of its internal pressure. This, in turn, delays the expansion and considerably prolongs the lifetime of the system. It was moreover shown [15] that this prolongation of the lifetime (as compared to the expansion of an ideal gas without transition) is in principle observable via an enhancement of the ratio of inverse widths, $R_{\text {out }} / R_{\text {side }}$, of the two-particle correlation function in out- and side-direction. (This signal was originally proposed by Pratt and Bertsch [16].) Another aspect [17,18] of the delayed expansion is the reduction of the transverse directed flow in semi-peripheral collisions that can be readily tested experimentally at fixed target energies [19].

In this paper I summarize the essential physics of the softening of the EoS in the transition region, and discuss as observable consequences the time-delayed expansion and the subsequent enhancement of $R_{\text {out }} / R_{\text {side, }}$, and the disappearance of the transverse directed flow. Natural units $\hbar=c=k_{B}=1$ are used throughout this paper.

\section{THE QCD PHASE TRANSITION AND SOFTENING OF THE EQUA- TION OF STATE}

Available lattice data for the entropy density in full QCD can be approximated by the simple parametrization [14,15,20]

$\frac{s}{s_{c}}(T)=\left[\frac{T}{T_{c}}\right]^{3}\left(1+\frac{d_{Q}-d_{H}}{d_{Q}+d_{H}} \tanh \left[\frac{T-T_{c}}{\Delta T}\right]\right)$,

where $s_{c}=$ const. $\times\left(d_{Q}+d_{H}\right) T_{c}^{3}$ is the entropy density at $T_{c}$. Pressure $p$ and energy density $\epsilon$ follow then from thermodynamical relationships. For $\Delta T=0$, the EoS (11) reduces to the MIT bag EoS 21] with bag constant $B=\frac{1}{2}\left(d_{Q} / d_{H}-1\right) T_{c} s_{c} /\left(d_{Q} / d_{H}+1\right)$. If one measures energies in units of $T_{c}$ and energy densities in units of the enthalpy density $\epsilon_{c}+p_{c}=T_{c} s_{c}$, the EoS (1) depends only on the ratio $d_{Q} / d_{H}$, and not on $d_{Q}$ and $d_{H}$ separately. For $\Delta T=0$, this ratio determines the latent heat (density) $\epsilon_{Q}-\epsilon_{H} \equiv 4 B$. (Here, $\epsilon_{Q}=\frac{1}{2}\left(4 d_{Q} / d_{H}-1\right) T_{c} s_{c} /\left(d_{Q} / d_{H}+1\right)$ is the energy density at the phase boundary between mixed phase and QGP, $\epsilon_{H}=\frac{3}{2} T_{c} s_{c} /\left(d_{Q} / d_{H}+1\right)$ is that at the boundary between mixed and hadronic phase.) 


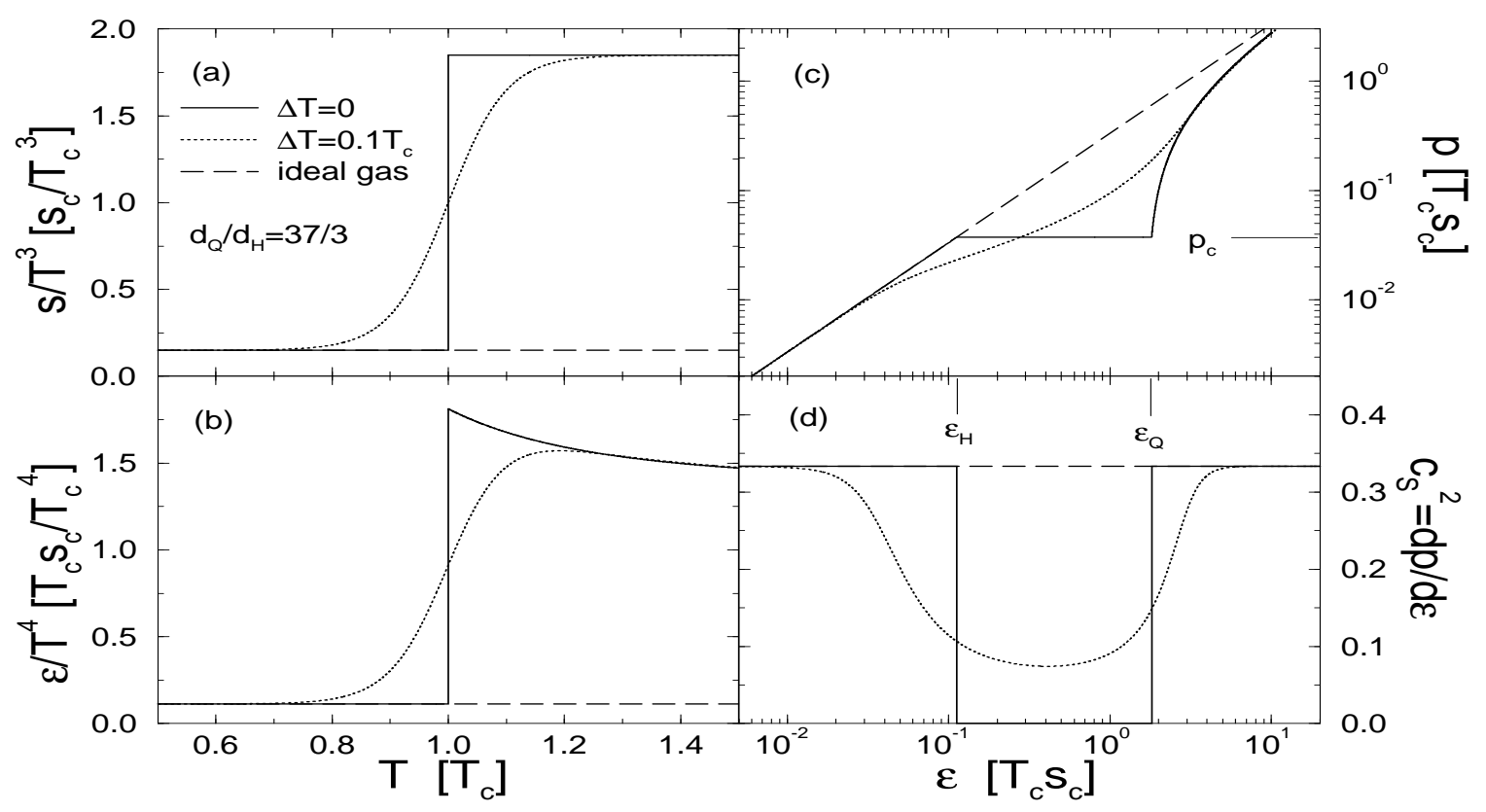

Figure 1. The equation of state: (a) entropy density divided by $T^{3}$, (b) energy density divided by $T^{4}$ as functions of temperature, (c) pressure, and (d) the velocity of sound squared as functions of energy density. Solid lines are for a first order transition $(\Delta T=0)$, dotted lines for a smooth transition with $\Delta T=0.1 T_{c}$, and dashed lines are for an ideal gas with $d_{H}$ degrees of freedom. The ratio of degrees of freedom is $d_{Q} / d_{H}=37 / 3$.

For the case $d_{H}=3$ (corresponding to an ultrarelativistic gas of pions) and $d_{Q}=37$ (corresponding to two massless flavours of quarks and antiquarks, and eight massless gluons), the latent heat, $4 B=1.7 T_{c} s_{c} \simeq 1.272 \mathrm{GeV} \mathrm{fm}^{-3}$, is large. On the other hand, including a resonance gas in the hadronic phase and/or reducing the effective number of degrees of freedom on the QGP side [22], $d_{Q} / d_{H}=3$ may be taken as a (perhaps more realistic) lower limit, with a smaller latent heat $\epsilon_{Q}-\epsilon_{H}=T_{c} s_{c}$. Assuming that the high-temperature phase consists of gluons only (such as expected for the "hot-glue scenario" [23) this would then correspond to about $400 \mathrm{MeV} \mathrm{fm}^{-3}$ in physical units. In the following, I shall focus on the case $d_{Q} / d_{H}=37 / 3$, for a discussion of $d_{Q} / d_{H}=3$, I refer to 15 .

Fig. 1 shows (a) the entropy density and (b) the energy density as functions of temperature, and (c) the pressure and (d) the velocity of sound squared $c_{S}^{2} \equiv \mathrm{d} p / \mathrm{d} \epsilon$ as functions of energy density for $\Delta T=0,0.1 T_{c}$, and an ideal gas with $d_{H}$ degrees of freedom for $d_{Q} / d_{H}=37 / 3$. Figs. 1 (a,b) present the thermodynamic functions in a form to facilitate comparison with lattice data. Present lattice data for full QCD can be approximated with a choice of $\Delta T$ in the range $0 \leq \Delta T<0.1 T_{c}$. In the hydrodynamical context, however, 
Figs. $1(\mathrm{c}, \mathrm{d})$ are more relevant. As can be seen in (c), for $\Delta T=0$ the pressure stays constant in the mixed phase $\epsilon_{H} \leq \epsilon \leq \epsilon_{Q}$. Hydrodynamical expansion is, however, driven by pressure gradients. It is therefore (the square of) the velocity of sound $c_{S}^{2}=\mathrm{d} p / \mathrm{d} \epsilon$, Fig. 1 (d), that is the most relevant measure of the system's tendency to expand. It represents the capability to perform mechanical work (which is proportional to pressure gradients $\mathrm{d} p$ ) for a given gradient in energy density $\mathrm{d} \epsilon$. For $\Delta T=0$, the velocity of sound vanishes in the mixed phase, i.e., mixed phase matter does not expand at all on its own account, even if there are strong gradients in the energy density. This has the consequence that it does not perform mechanical work and therefore cools less rapidly. For finite $\Delta T$, pressure gradients are finite, but still smaller than for an ideal gas EoS, and therefore the system's tendency to expand is also reduced, cf. Fig. 1 (d).

The reduction of $c_{S}^{2}$ in the transition region is commonly referred to as "softening" of the EoS, the respective region of energy densities is called "soft region" [12 15]. For matter passing through that region during the expansion phase, the flow will temporarily slow down or even possibly stall under suitable conditions and consequently lead to a time delay in the expansion of the system.

\section{HYDRODYNAMICS}

Hydrodynamics is defined by local energy-momentum conservation,

$\partial_{\mu} T^{\mu \nu}=0$.

Under the assumption of local thermodynamical equilibrium (the so-called "ideal fluid" approximation) the energy-momentum tensor $T^{\mu \nu}$ assumes the particularly simple form 24

$T^{\mu \nu}=(\epsilon+p) u^{\mu} u^{\nu}-p g^{\mu \nu}$,

where $u^{\mu}=\gamma(1, \mathbf{v})$ is the 4 -velocity of the fluid ( $\mathbf{v}$ is the 3 -velocity, $\gamma \equiv\left(1-\mathbf{v}^{2}\right)^{-1 / 2}$, $\left.u_{\mu} u^{\mu}=1\right)$, and $g^{\mu \nu}=\operatorname{diag}(+,-,-,-)$ is the metric tensor. The system of equations (2) is closed by choosing an EoS in the form $p=p(\epsilon)$, i.e., as depicted in Fig. 1 (c). In the ideal fluid approximation, the (equilibrium) EoS is the only input to the hydrodynamical equations of motion (2) that relates to properties of the matter under consideration and is thus able to influence the dynamical evolution of the system. The final results are uniquely determined once a particular initial condition and a decoupling ("freeze-out") hypersurface are specified.

For finite baryon density, one has to also take into account local conservation of baryon number,

$\partial_{\mu} N^{\mu}=0$

where $N^{\mu}=n u^{\mu}$ is the baryon 4 -current (in the ideal fluid approximation), $n$ is the baryon density in the local rest frame of a fluid element. In this case, the EoS has in general to be provided in the form $p=p(\epsilon, n)$ (see Fig. 1 of Ref. 18 for an explicit example).

Numerical methods to solve the hydrodynamical equations have been discussed for instance in [13]. In the following, I shall first discuss the time delay in the framework 


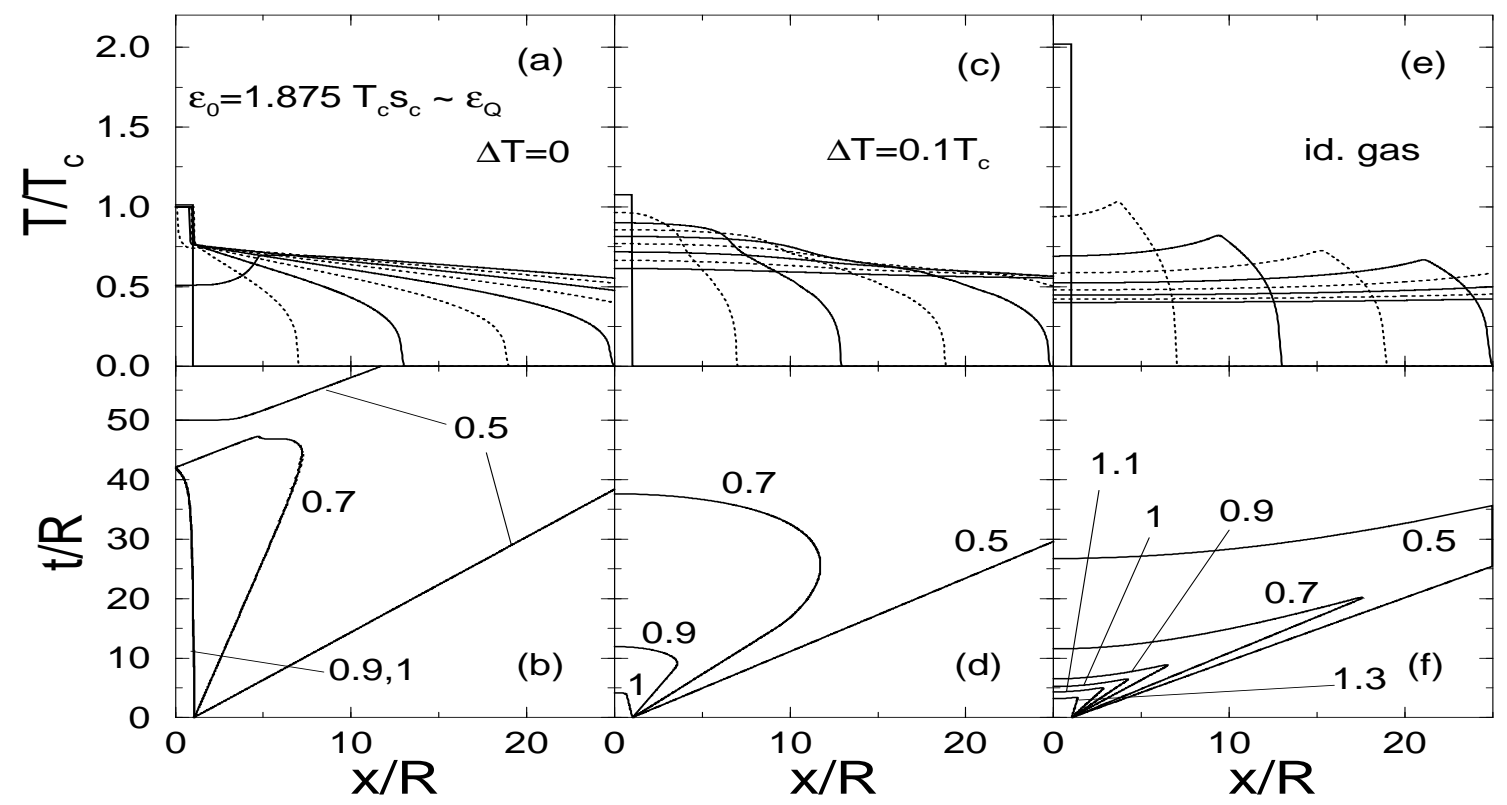

Figure 2. Longitudinal expansion in the Landau model: (a,c,e) temperature profiles for times $t=6 n \lambda R, \lambda=0.99, n=0,1,2, \ldots 8$ (alternatingly shown as full and dotted lines), (b,d,f) isotherms in the space-time diagram. (a,b) are for $\Delta T=0,(\mathrm{c}, \mathrm{d})$ for $\Delta T=0.1 T_{c}$, and $(e, f)$ for an ideal gas. The isotherms are labelled by the corresponding temperature in units of $T_{c}$.

of the simple Landau model [25], describing the one-dimensional expansion of a slab of matter (with infinite extension in transverse direction). I then consider time delay in the framework of the so-called Bjorken model [26], which is the three-dimensional expansion of a cylinder with boost-invariant initial conditions along its axis. This model is supposedly a good description of the expansion stage in ultrarelativistic heavy-ion collisions. Finally, I discuss the transverse directed flow for semi-peripheral $\mathrm{Au}+\mathrm{Au}$ collisions.

The Landau model requires to solve the hydrodynamical equations in time and only one space direction. Moreover, for the Bjorken model the cylindrical symmetry and longitudinal boost invariance reduce the equations of motion to a similar, effectively $1+1$ dimensional set of equations. To solve them I employ the relativistic Harten-Lax-van Leer-Einfeldt algorithm [28] tested in [13] and modified by a Sod predictor-corrector step to account for geometry and boost invariance in 15. The fully $3+1$ dimensional problem of a semi-peripheral $\mathrm{Au}+\mathrm{Au}$ collision is solved via operator splitting and the well-established SHASTA algorithm [13,29].

\section{DELAYED EXPANSION AND TWO-PARTICLE CORRELATIONS}

In this section I discuss the delayed expansion and observable consequences in the Landau [25] and Bjorken model [26] and for the EoS (11). Fig. 2 presents the hydrodynamic solutions for the purely $1+1$ dimensional expansion in the Landau model, for an initial 


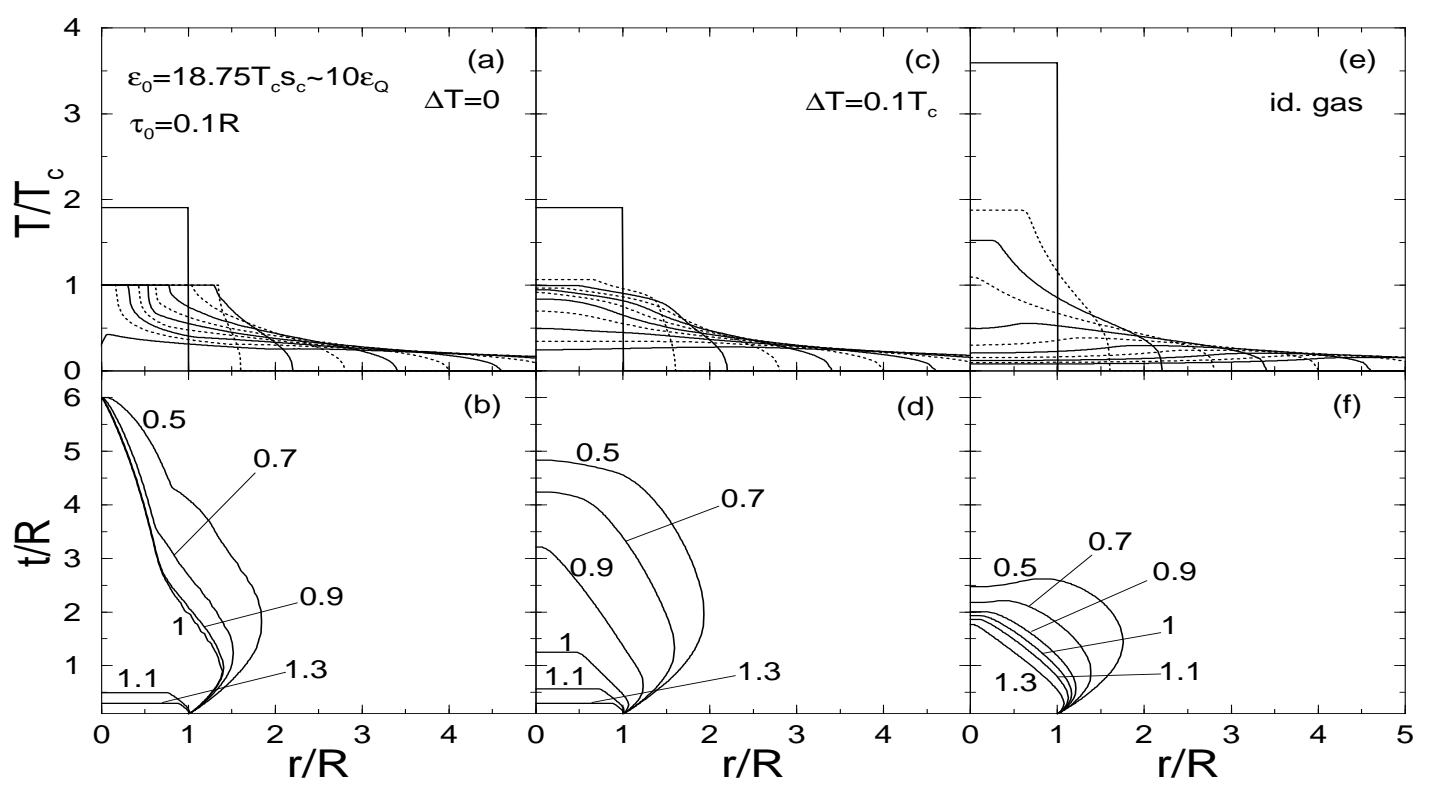

Figure 3. Transverse expansion in the Bjorken model. The presentation is analogous to Fig. 2, the initial energy density, however, is now ten times higher, $\epsilon_{0} \sim 10 \epsilon_{Q}$. The temperature profiles in (a,c,e) are shown for times $t=\tau_{0}+0.6 n \lambda R, \tau_{0}=0.1 R, \lambda=0.99$.

(homogeneously distributed) energy density $\epsilon_{0}=1.875 T_{c} s_{c} \sim \epsilon_{Q} \simeq 1.4 \mathrm{GeV} \mathrm{fm}^{-3}$. Figs. 2 (a,c,e) show temperature profiles as a function of $x$ (in units of half the initial extension of the slab, $R),(\mathrm{b}, \mathrm{d}, \mathrm{f})$ isotherms in the $t-x$ plane (labelled with the corresponding temperatures in units of $T_{c}$ ). As one observes, for a phase transition with a sharp first order transition $(\Delta T=0)$, Figs. $2(\mathrm{a}, \mathrm{b})$, or for a smooth transition $\left(\Delta T=0.1 T_{c}\right)$, Figs. $2(\mathrm{c}, \mathrm{d})$, the system stays hot (i.e., at temperatures around $0.7 T_{c}$ ) for a rather long time. The reason is, as explained in the preceding section, the softening of the EoS in the mixed phase which considerably reduces pressure gradients that, for instance, drive the expansion in the ideal gas case, Figs. 2 (e,f).

In addition, for $\Delta T=0$, the type of hydrodynamical expansion solution changes from an ordinary rarefaction wave to a rarefaction shock wave [13, 14, or so-called deflagration. That deflagration has a rather small propagation velocity for energy densities around $\epsilon_{Q}$ [30] and thus additionally prolongs the lifetime of the system. In this case, temperatures as high as $T_{c}$ persist for times as long as $42 R$. In the case $\Delta T=0.1 T_{c}$, such high temperatures vanish almost as fast as in the ideal gas case, but the softening of the EoS leads to a prolongation of the lifetime of temperatures around $0.7 T_{c}$ as compared to the ideal gas case. Thus, in view of the uncertainty in the QCD equation of state, it is rather unlikely that, as discussed in [12], enhanced electromagnetic radiation from a long-lived mixed phase is a viable signature for the transition to the QGP. On the other hand, if the system freezes out at sufficiently cool temperatures, the long lifetime of matter with $T \simeq 0.7 T_{c}$ could be observed via two-particle correlations. This idea shall be pursued further in the following discussion of the more realistic Bjorken expansion scenario.

The main assumption of Bjorken's model is longitudinal boost invariance which implies that the longitudinal flow velocity of matter is always given by $v^{z} \equiv z / t[26$ ]. The initial 


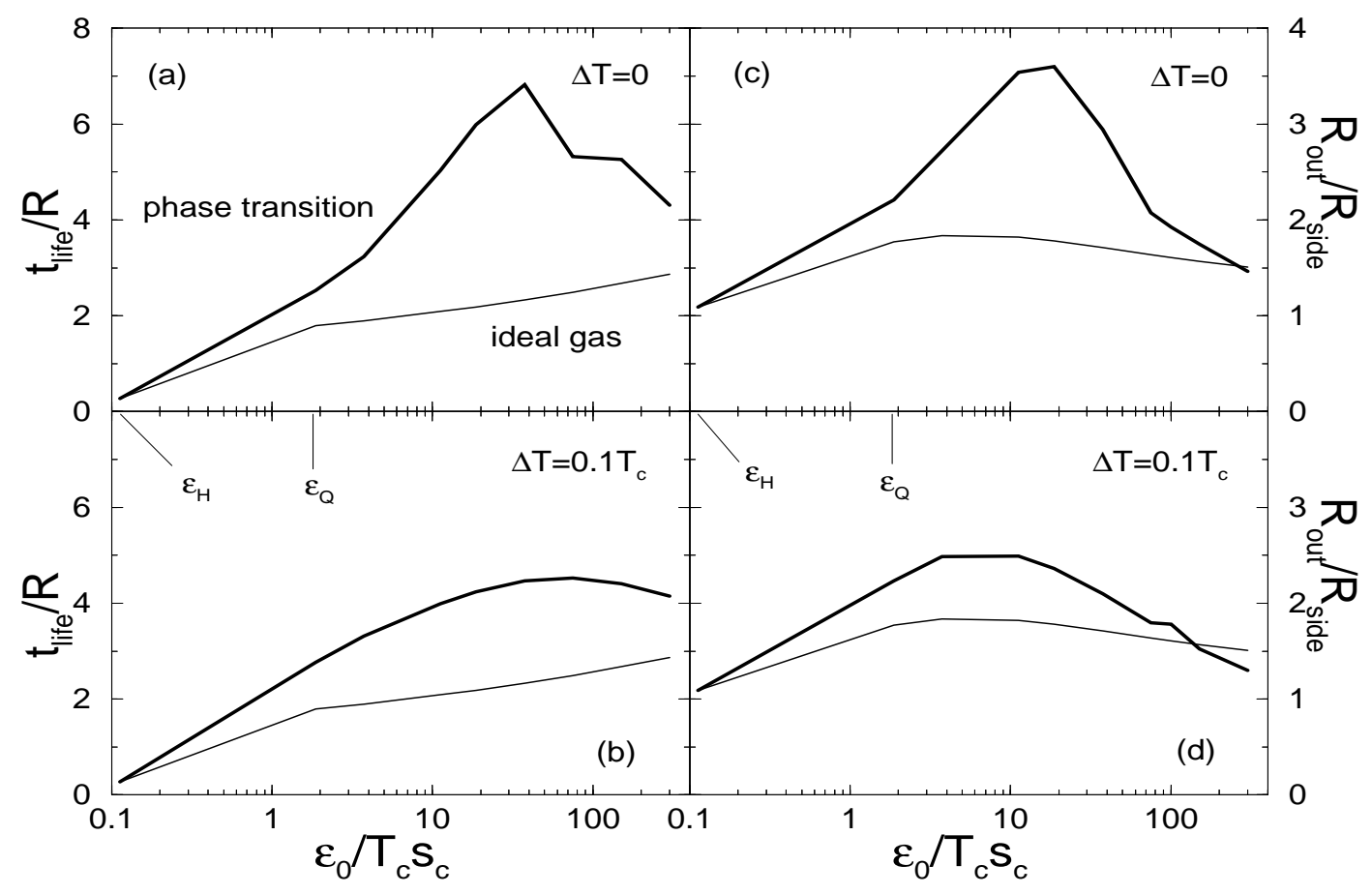

Figure 4. Lifetimes $(\mathrm{a}, \mathrm{b})$ and ratio of inverse widths of correlation functions $(\mathrm{c}, \mathrm{d})$ as a function of initial energy density for the Bjorken expansion. The thick lines correspond to the case of a transition to the QGP, the thin lines to the ideal gas expansion.

conditions are specified at constant proper time $\tau \equiv \sqrt{t^{2}-z^{2}}$. I fix $\tau_{0}=0.1 R$, motivated by the fact that for $\mathrm{Au}+\mathrm{Au}$ collisions at RHIC energies equilibration is expected [1]] to occur after $0.5 \mathrm{fm}$, while the initial radius $R$ of the hot zone is on the order of $5 \mathrm{fm}$. Fig. 3 shows hydrodynamic solutions for the (cylindrically symmetric) transverse expansion of a "Bjorken cylinder" (at $z=0$ ), for an initial energy density $\epsilon_{0}=18.75 T_{c} s_{c} \sim 10 \epsilon_{Q} \simeq 14$ $\mathrm{GeV} \mathrm{fm}^{-3}$. This value is expected to be reached through mini-jet production at RHIC energies [23]. Again, for a transition to the QGP the system spends considerable time in the "soft region" of the EoS (corresponding to temperatures around $T_{c}$ ), where pressure gradients are small, and therefore the expansion is delayed, Figs. $3(\mathrm{a}-\mathrm{d})$, in comparison to the ideal gas case, Figs. 3 (e,f). Note that this delay now occurs at about 10 times higher energy density than in the Landau model. This is due to the fact that the strong dilution due to the longitudinal velocity field has to be compensated so that the system stays long enough in the "soft" transition region.

Figs. $4(\mathrm{a}, \mathrm{b})$ show the lifetime [27] of matter with temperature $T=0.7 T_{c}$ as a function of initial energy density $\epsilon_{0}$ for the Bjorken expansion. One observes a distinguished maximum in the lifetime associated with the transition to the QGP. In accordance with the explanation provided above, this maximum of the lifetime occurs at energy densities above the "soft region" of the EoS. For systems with zero initial velocity, such as a spherically symmetric fireball at rest, the maximum would occur around $\epsilon_{0} \sim \epsilon_{Q}$ [14, 15].

Let us assume that the system decouples at $T=0.7 T_{c}$. Then, the isotherm with $T=0.7 T_{c}$ in Figs. $3(\mathrm{~b}, \mathrm{~d}, \mathrm{f})$ is the decoupling or "freeze-out" hypersurface. Once this 


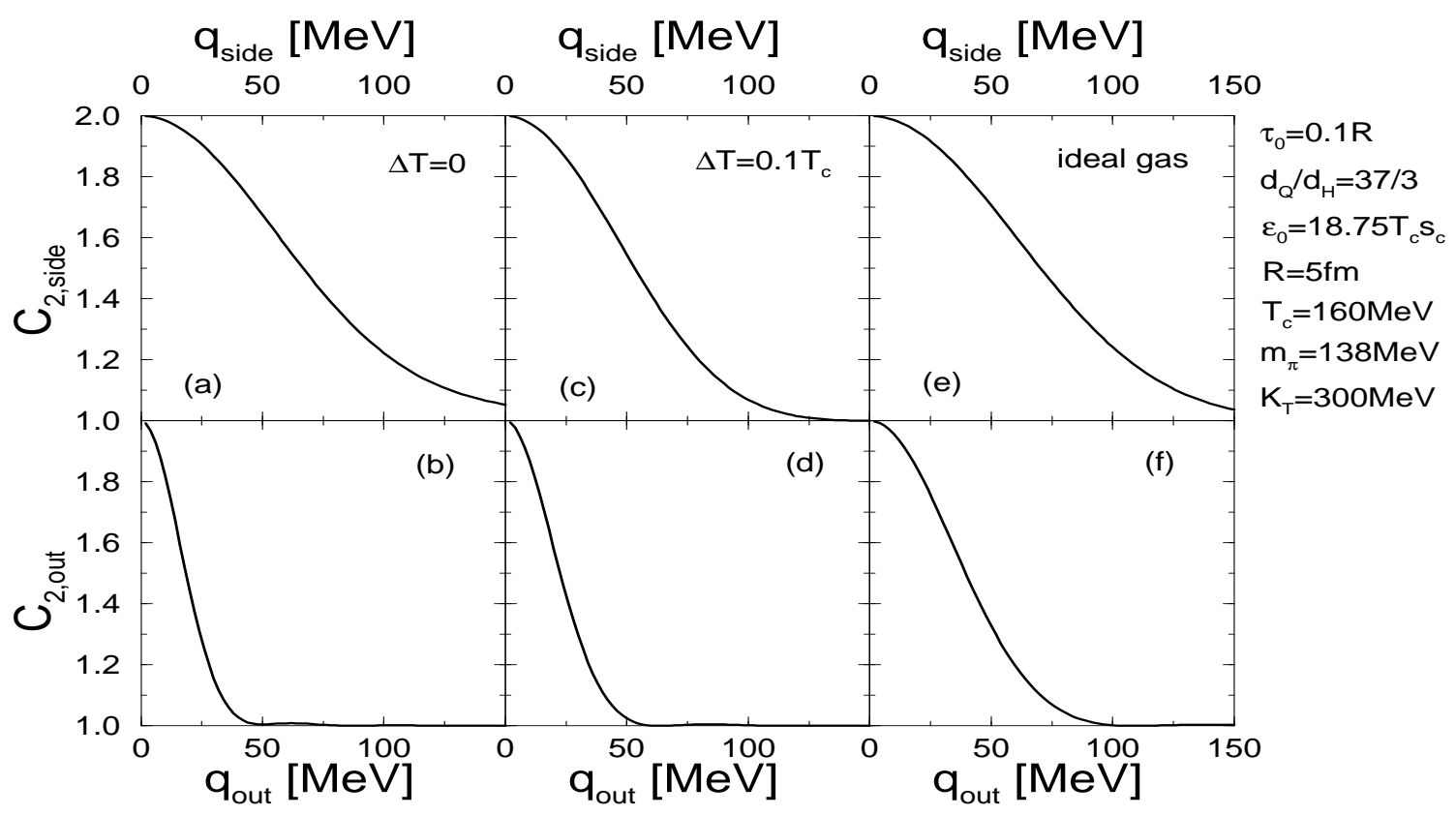

Figure 5. "Side" (a,c,e) and "out" correlation functions (b,d,f) calculated along the $T=$ $0.7 T_{c}$ isotherms of Figs. $3(\mathrm{~b}, \mathrm{~d}, \mathrm{f})$.

hypersurface is determined, one can also calculate the corresponding particle spectra. For single inclusive spectra, one commonly employs the formula of Cooper and Frye [31, and for two-particle correlation functions a suitable generalization [32]. In this manner, one can determine the two-pion correlation function $C_{2}\left(q_{\text {side }}, q_{\text {out }}, \mathbf{K}\right)$ as a function of the relative pion momenta $q_{\text {out }}$ or $q_{\text {side }}$ for fixed average momentum $\mathbf{K}$.

For fixed $\mathbf{K}$ one defines the so-called "side" correlation function as $C_{2 \text {,side }}\left(q_{\text {side }}\right) \equiv$ $C_{2}\left(q_{\text {side }}, 0, \mathbf{K}\right)$, and the "out" correlation function as $C_{2 \text {,out }}\left(q_{\text {out }}\right) \equiv C_{2}\left(0, q_{\text {out }}, \mathbf{K}\right)$. The inverse width of the "out" correlation function is a measure for the duration of particle emission, i.e., the lifetime of the system, while the inverse width of the "side" correlation function measures its transverse size [16].

Fig. 5 shows these functions for $(\mathrm{a}, \mathrm{b})$ the case of a sharp first order transition $\Delta T=0$, (c,d) a smooth transition $\Delta T=0.1 T_{c}$, and $(\mathrm{e}, \mathrm{f})$ the ideal gas expansion. The correlation functions are calculated along the $T=0.7 T_{c}$ isotherms of Figs. $3(\mathrm{~b}, \mathrm{~d}, \mathrm{f})$ with $\mathbf{K}=$ $(K, 0,0), K=300 \mathrm{MeV}$, and $R=5 \mathrm{fm}$ to fix the $q$-scale. The pion mass is assumed to be $m_{\pi}=138 \mathrm{MeV}$, and $T_{c}=160 \mathrm{MeV}$. Note that the long lifetime of the system in Fig. 3 (b) is reflected in the small width of the corresponding "out" correlation function in Fig. 5 (b), while the similar transverse size in all three cases leads to rather similar "side" correlation functions.

Given the correlation functions, one then determines the ratio of inverse widths, $R_{\text {out }} /$ $R_{\text {side }}$. (The width of the correlation function is here taken as the $q$-value where $C_{2}=1.5$.) If the systems have similar transverse size, one then expects that this ratio is a good measure for the lifetime of the system. Figs. 4 (c,d) show $R_{\text {out }} / R_{\text {side }}$ as a function of the initial energy density $\epsilon_{0}$. As expected, this ratio mirrors closely the dependence of 
the lifetime on initial conditions in Figs. 4 (a,b). The effect is maximized around initial energy densities expected to be reached at the RHIC collider [23]. The enhancement over the ideal gas case is of the order of $40-100 \%$ (for $\Delta T=0.1 T_{c}$ to $\Delta T=0$ ).

At energy densities estimated to be reached in CERN SPS Pb+Pb collisions $\left(\epsilon_{0} \sim\right.$ $1-2 T_{c} s_{c}$ in our units), one expects from the above that $R_{\text {out }} / R_{\text {side }} \sim 1.5-2$. However, present data from CERN SPS [33] indicate that the (fitted) out-radii are rather similar to the side-radii. This does not contradict my results, because, as shown by Schlei et al. [34] in the framework of a hydrodynamical calculation similar to mine, correlation functions constructed from thermal pions only give $R_{\text {out }} / R_{\text {side }} \sim 2$ (cf. especially [32]), while the incorporation of long-lived resonance decays leads to a reduction of that ratio and good agreement with the measured radii. Note that kaon interferometry 35 37] is preferable, though experimentally more difficult, because only distortions of the interference pattern due to shorter lived $K^{*}$ resonances have to be taken into account.

\section{DIS- AND REAPPEARANCE OF TRANSVERSE DIRECTED FLOW}

The softening of the EoS and the delay in the expansion have an interesting consequence for semi-peripheral heavy-ion collisions at AGS energies. If the hot, compressed (baryonrich) matter in the central zone undergoes a transition to the QGP, its tendency to expand is reduced, similarly as discussed above. This prevents the deflection of spectator matter, as it would occur for a "stiff" EoS with a stronger tendency to expand, for instance a purely hadronic EoS without phase transition [18]. As shown in Fig. 6, this effect is observable in the excitation function of the transverse directed flow per baryon,

$\left\langle p_{x} / N\right\rangle^{d i r}=\frac{1}{N} \int_{-y_{C M}}^{y_{C M}} \mathrm{~d} y\left\langle p_{x} / N\right\rangle(y) \frac{\mathrm{d} N}{\mathrm{~d} y} \operatorname{sgn}(y)$

The average transverse momentum per nucleon for a given (fluid) rapidity $y$ is here defined as $\left\langle p_{x} / N\right\rangle(y)=m_{N}\left\langle v_{x}\right\rangle\langle\gamma\rangle$, where $m_{N}=938 \mathrm{MeV},\langle\gamma\rangle \equiv\left(1-\sum_{i}\left\langle v_{i}\right\rangle^{2}\right)^{-1 / 2}$, and

$\left\langle v_{i}\right\rangle \equiv \frac{1}{\Delta N} \int_{-y-\Delta y / 2}^{y+\Delta y / 2} \mathrm{~d} y v_{i} \frac{\mathrm{d} N}{\mathrm{~d} y}, i=x, y, z$

$\Delta N=\int_{-y-\Delta y / 2}^{y+\Delta y / 2} \mathrm{~d} y \mathrm{~d} N / \mathrm{d} y$

The overall decrease of this quantity above $E_{\mathrm{Lab}}^{\mathrm{kin}} \sim 2 \mathrm{AGeV}$ observed for both EoS is simply due to the fact that faster spectators are less easily deflected by the hot, expanding participant matter. One clearly observes a dramatic drop between BEVALAC and AGS beam energies and an increase beyond $\sim 10 \mathrm{AGeV}$ for the EoS with phase transition as compared to the calculation with the pure hadronic EoS. Thus, there is a local minimum in the excitation function of the directed transverse (in-reaction-plane) collective flow around $\sim 6 \mathrm{AGeV}$, which is again related to the phase transition to the QGP and the existence of a "soft region" in the nuclear matter EoS. Note that the position of the minimum strongly depends on the details of the EoS. It may easily shift to higher beam energies, if more resonances are included in the hadronic part of the EoS. Also, absolute values for the directed flow cannot yet be compared to experimentally measured ones, since at this stage freeze-out has not been performed. Moreover, (physical) viscosity is neglected in the ideal hydrodynamic picture, which is known to have a strong influence on flow 


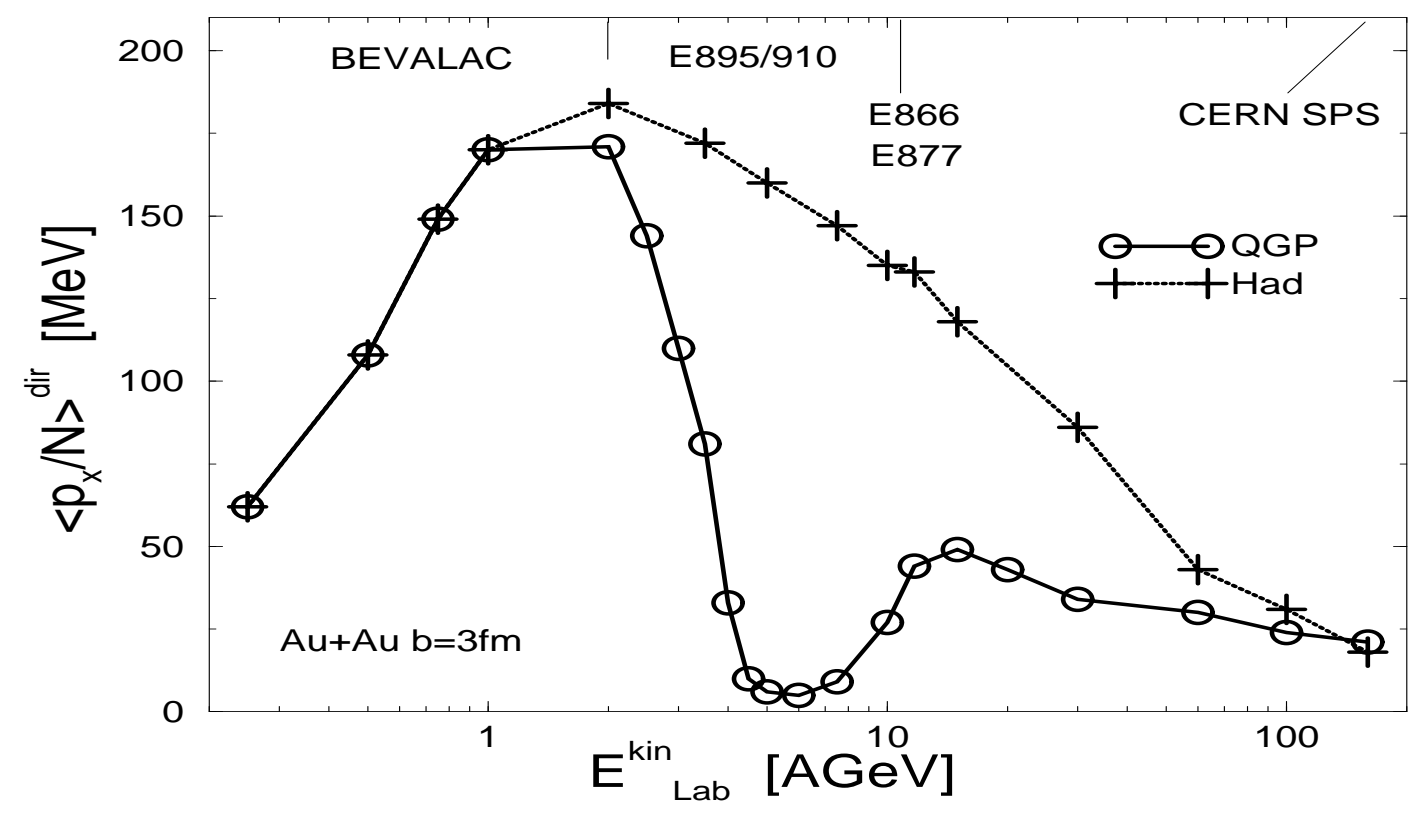

Figure 6 . The excitation function of the transverse directed flow as calculated from $3+1$ dimensional hydrodynamics for $\mathrm{Au}+\mathrm{Au}$ collisions at impact parameter $b=3 \mathrm{fm}$. The full line (and the circles) is for an EoS with phase transition to the QGP, the dotted curve (and the crosses) is for a purely hadronic EoS.

[38]. On the other hand, there is a certain amount of numerical viscosity inherent in the transport scheme that solves the hydrodynamical equations. This viscosity must be large enough to suppress numerical instabilities 13,39, but it will in turn also affect the position of the minimum [40]. Finally, the nature of the transition at finite baryon density is completely unknown. In the above calculation, a sharp first order transition was assumed [18]. A smooth transition (similar to that in Fig. 1) would certainly tend to wash out the minimum in the excitation function of the flow, as pressure gradients become bigger and increase the system's tendency to expand and deflect spectator matter. The main point is, however, that irrespective of these quantitative uncertainties, the minimum is a generic qualitative signal for a transition from hadron to quark and gluon degrees of freedom in the nuclear matter EoS.

A more realistic description of heavy-ion collisions, especially for higher bombarding energies, can be achieved with the so-called three-fluid model [41]. Ordinary one-fluid dynamics assumes instantaneous local equilibrium, even in the initial collision stage. This leads to instantaneous stopping of target and projectile, which is obviously unrealistic since the stopping power of nuclear matter is finite. In the three-fluid approach, one solves this problem by assuming that target and projectile are separate fluids which interact via collision terms derived from kinetic theory. Collisions among target and projectile fluid elements create a third fluid. Fig. 7 shows a calculation of $\left\langle p_{x} / N\right\rangle(y)$ for a semi-peripheral $(b=4.5 \mathrm{fm}) \mathrm{Pb}+\mathrm{Pb}$ collision at $11 \mathrm{AGeV}$. Since this is an ongoing investigation, I am yet not able to show results for the excitation function of the transverse directed flow. 


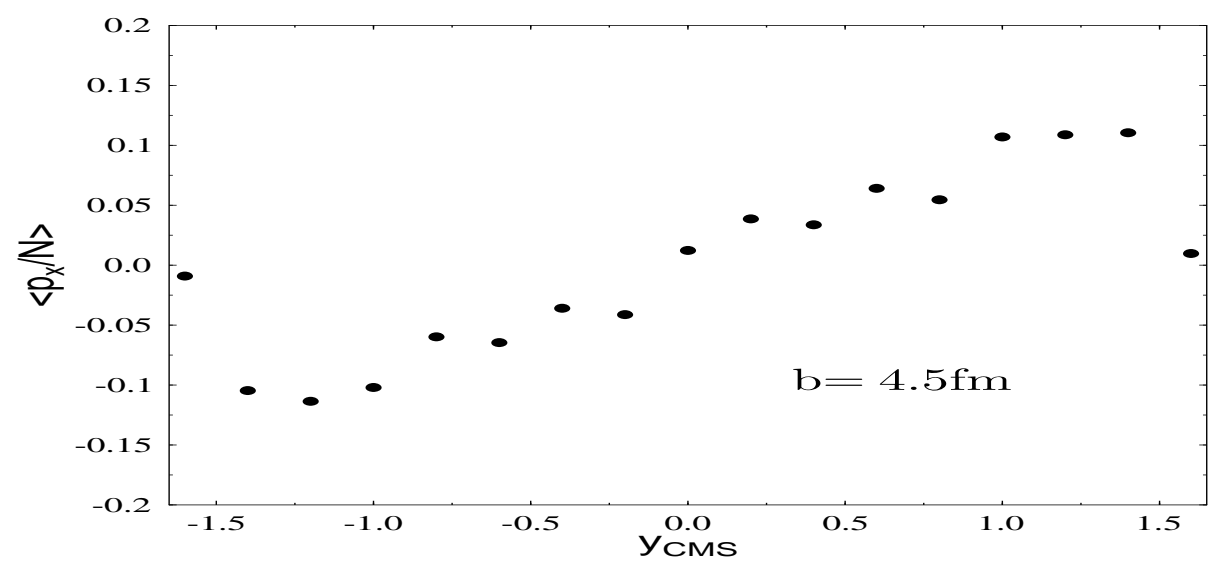

Figure 7. The transverse momentum as a function of rapidity as calculated from $3+1$ dimensional three -fluid dynamics for a $\mathrm{Pb}+\mathrm{Pb}$ collision at $11 \mathrm{AGeV}$ and impact parameter $b=4.5 \mathrm{fm}$.

\section{CONCLUSIONS}

In this paper I have discussed the softening of the nuclear matter EoS due to a transition to the QGP. Since present lattice data only constrain the width of the transition region to be in the range $0 \leq \Delta T<0.1 T_{c}$, it is important to test how such uncertainties may influence dynamical observables. This has to be investigated in the framework of relativistic hydrodynamics, since that is the only model that provides a direct link between the EoS and collective observables.

I focussed on the lifetime of the system as a function of initial energy density as one possible collective observable that can discriminate between different EoS. One-dimensional studies in the framework of the Landau expansion model [14] show that the lifetime is much longer in the case of a first order phase transition, $\Delta T=0$, as compared to the expansion of an ideal gas without transition. The prolongation of the lifetime can be up to a factor of 10, provided the initial energy density corresponds to that of mixed phase with a large fraction of QGP. In that case, rarefaction proceeds as a (slow) deflagration shock wave instead of a simple rarefaction wave (with the speed of sound as characteristic propagation velocity). In the case of a smooth transition with $\Delta T=0.1 T_{c}$, deflagration solutions no longer exist, but due to the reduction of the velocity of sound in the transition region the expansion is still slower than that of an ideal gas. Thus, the lifetimes (of matter with $T=0.7 T_{c}$ ) remain on the order of a factor of 7 longer as compared to the ideal gas expansion. 
The results for the Bjorken cylinder expansion [15] were similar as for the Landau expansion, up to two important exceptions: (a) the maximum lifetimes emerged at higher initial energy densities corresponding to QGP matter instead of mixed phase matter, and (b) the lifetimes were in general shorter. Both effects are explained by the very efficient cooling due to the initial longitudinal velocity profile associated with the boost invariance of the problem. This effect causes an overall reduction of the lifetimes. Moreover, in order to remain long enough in the soft region of the EoS and thus to slow down (or even stall) the cooling process in transverse direction, one has to start at higher initial energy densities to compensate for the longitudinal cooling. Otherwise, the longitudinal cooling reduces the energy densities too fast and one leaves the soft region of the EoS too soon. Then, the expansion and cooling is accelerated due to the higher velocity of sound outside the soft region of the EoS.

I then showed (cf. also [16]) that the prolongation of the lifetime can be observable via the ratio $R_{\text {out }} / R_{\text {side }}$ of inverse widths of two-particle correlation functions in outand side-direction. This ratio follows the behaviour of the lifetimes rather closely. The prolongation of the lifetime in the case of a transition to the QGP could therefore be in principle searched for using this observable. The enhancement of that ratio is, of course, strongest in the case that the transition is first order with a large latent heat. An interesting result is that, for the Bjorken cylinder geometry, the maximum of $R_{\text {out }} / R_{\text {side }}$ occurs at energy densities presumably reached at the RHIC collider.

There are several effects which may reduce the strength of the time-delay signal observable via the $R_{\text {out }} / R_{\text {side }}$ ratios that will require further investigation. First, the decay of long-lived resonances can simulate time delay [42]. Interferometry with kaons instead of pions is therefore preferable [37]. Finally, while the present investigations covered a wide range of uncertainties in the EoS, the calculations have neglected effects of dissipation that tend in general to reduce the collective flow strengths predicted via ideal hydrodynamics. For instance, bulk viscosity appears in the hydrodynamical equations of motion in a similar way as the pressure, and could in principle counteract any reduction of the velocity of sound in the transition region. The main result of this paper is, nevertheless, that the generic time-delay signature of QGP formation is remarkable robust to present uncertainties in the QCD equation of state.

I finally discussed the dis- and reappearance of the transverse directed flow around AGS energies. As a result of the softening of the EoS in the transition region, the tendency for the hot and dense reaction zone to expand is considerably reduced. Thus, spectator matter passes this zone undeflected, which consequently leads to a substantial decrease in the transverse flow as compared to a purely hadronic scenario without transition to the QGP. Quantitative estimates for the size of this effect are subject to large uncertainties, as it appears to be sensitive to the EoS used, and is influenced by numerical and (so far neglected) physical viscosity. Also, freeze-out has not yet been performed to calculate the particle (instead of fluid) flow. The local minimum in the excitation function of the transverse directed flow remains, however, an important qualitative signal for a transition (to the QGP) in the nuclear matter EoS. 


\section{Acknowledgments}

I would like to thank the organizers for their invitation to present these results at Quark Matter '96. I am indebted to G. Bertsch, U. Heinz, D. Keane, B. Schlei, E. Shuryak, and W. Zajc for valuable comments and discussions on time delay and interferometry. Special thanks go to J. Brachmann and A. Dumitru for their efforts to provide me with Figure 7, and to S. Bernard for discussions related to $1+1$ dimensional transport algorithms. Finally, I am most grateful to Miklos Gyulassy for his continuing interest and his encouragement to pursue this subject, for discussions, and for all other kinds of support during my time at Columbia University.

\section{REFERENCES}

1. see, for instance, the contribution of E. Laermann in these proceedings.

2. see, for instance:

S.A. Chin, Phys. Lett. B 119 (1982) 51,

L.D. McLerran and T. Toimela, Phys. Rev. D 31 (1985) 545,

R.C. Hwa and K. Kajantie, Phys. Rev. D 32 (1985) 1109,

K. Kajantie, J. Kapusta, L. McLerran, A. Mekjian, Phys. Rev. D 34 (1986) 2746.

3. T. Matsui and H. Satz, Phys. Lett. B 178 (1986) 416.

4. M. Gyulassy and M. Plümer, Phys. Lett. B 243 (1990) 432.

5. C. Greiner, D.H. Rischke, H. Stöcker, P. Koch, Phys. Rev. D 38 (1988) 2797.

6. K. Rajagopal and F. Wilczek, Nucl. Phys. B 399 (1993) 395.

7. J. Kapusta, P. Lichard, D. Seibert, Phys. Rev. D 44 (1991) 2774.

8. Renewed interest in that signature, has, however, emerged with the advent of recent results from the NA50 experiment at the CERN SPS, showing a rapid variation of $J / \Psi$-suppression as a function of transverse energy in $\mathrm{Pb}+\mathrm{Pb}$ collisions at $158 \mathrm{AGeV}$, see the contribution of M. Gonin in these proceedings.

9. C. Gerschel and J. Hüfner, Nucl. Phys. A 544 (1992) 513c.

10. For a review, see:

H. Stöcker and W. Greiner, Phys. Rep. 137 (1986) 277,

R.B. Clare and D.D. Strottman, Phys. Rep. 141 (1986) 177.

11. X.-N. Wang, M. Gyulassy, M. Plümer, Phys. Rev. D 51 (1995) 3436,

R. Baier, Yu.L. Dokshitzer, S. Peigné, D. Schiff, Phys. Lett. B 345 (1995) 277,

R. Baier, Yu.L. Dokshitzer, A.H. Mueller, S. Peigné, D. Schiff, preprint CERN-TH96/14, 1996 (unpublished).

12. C.M. Hung and E.V. Shuryak, Phys. Rev. Lett. 75 (1995) 4003.

13. D.H. Rischke, S. Bernard, J.A. Maruhn, Nucl. Phys. A 595 (1995) 346.

14. D.H. Rischke and M. Gyulassy, Nucl. Phys. A 597 (1996) 701.

15. D.H. Rischke and M. Gyulassy, Nucl. Phys. A (in press).

16. S. Pratt, Phys. Rev. C 49 (1994) 2722, Phys. Rev. D 33 (1986) 1314,

G. Bertsch, Nucl. Phys. A 498 (1989) 173c.

17. L.V. Bravina, N.S. Amelin, L.P. Csernai, P. Levai, D. Strottman, Nucl. Phys. A 566 (1994) 461c.

18. D.H. Rischke, Y. Pürsün, J.A. Maruhn, H. Stöcker, W. Greiner, preprint CU-TP-695, 
nucl-th/9505014 (to be published in Heavy Ion Phys.).

19. J. Barrette et al. (E877 collaboration), Phys. Rev. Lett. 73 (1994) 2532, Nucl. Phys. A 590 (1995) 259c,

Y. Zhang and J.P. Wessels (E877 collaboration), Nucl. Phys. A 590 (1995) 557c,

G. Rai and the E895 collaboration, LBL PUB-5399 (1993).

20. J.P. Blaizot and J.Y. Ollitrault, Phys. Rev. D 36 (1987) 916.

21. A. Chodos, R.L. Jaffe, K. Johnson, C.B. Thorn, V.F. Weisskopf, Phys. Rev. D 9 (1974) 3471.

22. J.J. Neumann, D. Seibert, G. Fai, Phys. Rev. C 51 (1995) 1460.

23. E. Shuryak, Phys. Rev. Lett. 68 (1992) 3270,

K.J. Eskola and M. Gyulassy, Phys. Rev. C 47 (1993) 2329.

24. L.D. Landau and E.M. Lifshitz, "Fluid mechanics" (Pergamon, New York, 1959).

25. L.D. Landau, Izv. Akd. Nauk SSSR 17 (1953) 51, in: "Collected papers of L.D. Landau" (ed. D. Ter-Haar, Pergamon, Oxford, 1965), p. 569-585,

L.D. Landau and S.Z. Belenkii, Uspekhi Fiz. Nauk 56 (1955) 309, in: "Collected papers of L.D. Landau" (ed. D. Ter-Haar, Pergamon, Oxford, 1965), p. 665-700.

26. J.D. Bjorken, Phys. Rev. D 27 (1983) 140,

G. Baym, B.L. Friman, J.P. Blaizot, M. Soyeur, W. Czyż, Nucl. Phys. A 407 (1983) 541.

27. Here defined as the intercept of an isotherm in Figs. 3 (b,d,f) with the $t$-axis.

28. V. Schneider et al., J. Comput. Phys. 105 (1993) 92.

29. J.P. Boris and D.L. Book, J. Comput. Phys. 11 (1973) 38,

D.L. Book, J.P. Boris, K. Hain, J. Comput. Phys. 18 (1975) 248.

30. L. Van Hove, Z. Phys. C 21 (1983) 93,

M. Gyulassy, K. Kajantie, H. Kurki-Suonio, L. McLerran, Nucl. Phys. B 237 (1984) 477.

31. F. Cooper and G. Frye, Phys. Rev. D 10 (1974) 186,

F. Cooper, G. Frye, E. Schonberg, Phys. Rev. D 11 (1975) 192.

32. B.R. Schlei, U. Ornik, M. Plümer, R.M. Weiner, Phys. Lett. B 293 (1992) 275.

33. B.V. Jacak (NA44 collaboration), Nucl. Phys. A 590 (1995) 215c.

34. J. Bolz, U. Ornik, M. Plümer, B.R. Schlei, R.M. Weiner, Phys. Rev. D 47 (1993) 3860 ,

B. Schlei, U. Ornik, M. Plümer, D. Strottman, R.M. Weiner, Los Alamos preprint hep-ph/9509426.

35. M. Gyulassy and S.S. Padula, Phys. Rev. C 41 (1990) 21.

36. B.R. Schlei, Los Alamos preprint nucl-th/9605016.

37. S. Bernard, D.H. Rischke, M. Gyulassy, in preparation.

38. W. Schmidt, U. Katscher, B. Waldhauser, J.A. Maruhn, H. Stöcker, W. Greiner, Phys. Rev. C 47 (1993) 2782.

39. D.H. Rischke, Y. Pürsün, J.A. Maruhn, Nucl. Phys. A 595 (1995) 383.

40. D.H. Rischke, in preparation.

41. A. Dumitru et al., Phys. Rev. C 51 (1995) 2166.

42. S.S. Padula and M. Gyulassy, Nucl. Phys. A 544 (1992) 537c. 